\title{
A Resource-Adaptive Mobile Navigation System
}

Jörg Baus

\author{
Antonio Krüger \\ Department of Computer Science, Saarland University \\ P.O. Box 1511 50, 66041 Saarbrücken, Germany \\ \{baus, krueger,wahlster\}@cs.uni-sb.de
}

\author{
Wolfgang Wahlster
}

\begin{abstract}
The design of mobile navigation systems adapting to limited resources will be an important future challenge. Since typically several different means of transportation have to be combined in order to reach a destination, the user interface of such a system has to adapt to the user's changing situation. This applies especially to the alternating use of different technologies to detect the user's position, which should be as seamless as possible. This article presents a hybrid navigation system that relies on different technologies to determine the user's location and that adapts the presentation of route directions to the limited technical resources of the output device and the limited cognitive resources of the user.
\end{abstract}

\section{Keywords}

mobile navigation, resource adaptivity, user adaptivity

\section{INTRODUCTION}

Personal navigation systems that extend beyond today's use in cars will play a major role in the future. Especially pedestrians will benefit from getting localized information on mobile devices anywhere at any time. Quite frequently, several different means of transportation have to be combined to reach a destination. When a change of the means of transportation is detected, the system has to adapt its interface to the new situative constraints. One essential issue in this context is the switch between different positioning technologies, e. g., from outdoor systems such as GPS (Global Positioning System) or the cell-based GSM (Global system for mobile communication)/UMTS (Universal Mobile Telecommunications System) to indoor systems such as infrared or Bluetooth and vice versa. Ideally, a system should conceal the switching between these technologies from the user entirely. In addition the presentation of way description has to be adapted to different output devices that may be used. This includes adaptations to the screen size, resolution, and color capabilities of mobile and stationary devices.

The precision of the positional information is subject to fluctuations that depend on the visibility of GPS satellites in the

\footnotetext{
Permission to make digital or hard copies of all or part of this work for personal or classroom use is granted without fee provided that copies are not made or distributed for profit or commercial advantage and that copies bear this notice and the full citation on the first page. To copy otherwise, or republish, to post on servers or to redistribute to lists, requires prior specific permission and/or a fee.

IUI'02, January 13-16, 2002, San Francisco, California, USA.

Copyright 2002 ACM 1-58113-459-2/02/0001...\$5.00
}

outdoor setting and on cell size (radio, bluetooth, and infrared) for indoor scenarios. A mobile pedestrian navigation system has to be able to react appropriately to varying quality and precision of the resulting measurements. Even more difficult than the adaptation to technical constraints is the adaptation to the cognitive limitations of the user for a given situation. Therefore, the focus of our research in the project REAL $^{1}$ was to investigate how a mobile pedestrian navigation system can adapt a multi-modal way description according to the detected limited technical and cognitive resources.

In terms of cognitive resources we take into account the user's actual traveling speed, her familiarity with the environment, and the time pressure in the current situation. Special adaptation services have to be provided in the case that navigation is not the user's primary goal. Additional tasks that have to be performed in parallel such as carrying heavy luggage or answering a telephone call while walking yield additional stress that has to be compensated by the system. REAL already attempts to achieve this during the process of way finding. Instead of choosing the shortest route, REAL tries to avoid complex instructions at the cost of a slightly longer route, thus minimizing the additional cognitive load on the user.

The remainder of this article is organized as follows. We first introduce our notion of resource and location sensitivity, and the systems architecture. We then give a brief review of related work in the area of mobile computing. In the following section we present our mobile navigation system and finally we summarize our findings and give an outlook on our future work.

\section{RESOURCE AND LOCATION SENSITIVITY}

We distinguish three different classes of resource sensitive processes: (a) resource adapted processes, (b) resource adaptive processes and (c) resource adapting processes [13]. Resource adapted processes have been optimized in advance for restricted resources that are well known and follow regular patterns. The quality of their results remains constant for a given input. In contrast resource adaptive and resource adapting processes can handle varying resource restrictions. Therefore, their results depend on the available resources during runtime. Resource adaptive processes rely on a sin-

\footnotetext{
${ }^{1}$ REAL is part of the Collaborative Research Center 378 on resourceadaptive cognitive processes
} 
gle strategy to react to varying resources, whereas resource adapting processes select among a number of strategies on a meta cognitive level to comply with different resource situations. This means that under certain conditions several strategies are used to solve a given problem. REAL uses both resource adaptive and resource adapting processes to generate way descriptions.

Furthermore, we discriminate two different kinds of location sensitivity for mobile devices: Active location sensitivity on the one hand implies that the mobile device actively detects the actual location on its own. Based on that information the presentation is generated automatically to comply with the user's requests. A typical example are GPS based car navigation systems. Passive location sensitivity on the other hand requires the mobile device to passively present the information that it receives from senders in its local environment, e. g., location based information on traffic status via Radio Data Service (RDS).

Active location sensitivity delegates most of the computational burden to the mobile device. Due to the fact that its memory and CPU performance are limited, the adaptation process becomes even more difficult. In contrast to that, passive location sensitivity relocates nearly all of the necessary computations to the instrumented environment. The mobile device reacts to changing situations by presenting localized information, which means information especially prepared for the actual location. In other words one could say that, the intelligence needed for active location sensitive processes has to be provided by the mobile device, whereas processes that rely on passive location sensitivity use the computational resources of the environment. Therefore, it is desirable to use passive location sensitivity in scenarios with many users and complex adaptation services, and active location sensitivity in scenarios with less users and limited adaption services.

In the project REAL we have developed a pedestrian navigation system that combines active and passive location sensitivity for the first time. The system has been designed in such a way that the change-over between both adaptation paradigms is barely noticeable for the user. REAL integrates two subsystems: the system IRREAL (Infrared REAL) for indoor navigation tasks based on infrared senders, and the system ARREAL (Augmented Reality REAL) for outdoor navigation based on GPS-satellites. IRREAL uses passive location sensitivity, whereas ARREAL relies on the active counterpart. The overall system architecture of REAL is shown in figure 1 . On the input level a request for a route description is transformed into a user specific request, taking into account limited cognitive and technical resources. The user specific request is then passed to the resource adaptive way finding module. With the help of domain knowledge and a user model, the optimal route is determined and forwarded to the presentation planning module. This module optimizes the presentation of the way description not only according to the resolution, screen size, and color capabilities of the

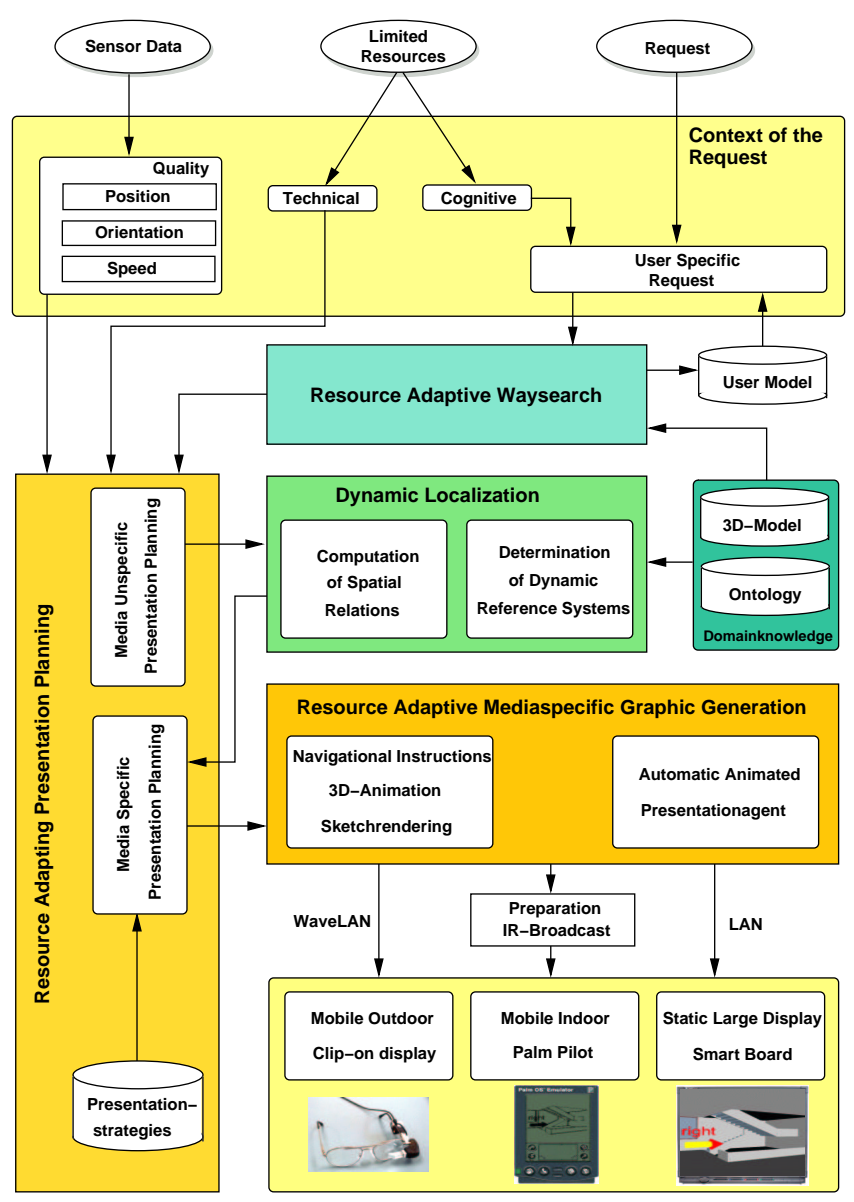

Figure 1: The system architecture of the hybrid navigation aid

output device, but also to the quality of the given sensor information (i. e. precision of location, orientation and speed of the user). The planning process consists of a media independent and a media dependent part. The media independent component relies on a 3D-model and an ontology of the environment. In a first step appropriate parts of the 3D-model are selected for visualization depending on the actual sensor quality [1]. Thereafter, the component evaluates spatial relations taking into account the results of experimental studies $[12,16]$. These relations are used to enhance the description according to the specific situation. This information is then passed to the media dependent planning component, which relies on design presentation strategies to adapt the way description to different output media.

This approach enables a single centralized server to produce all presentations. At the moment the system can adapt its presentations to a large screen (e.g., a Smartboard), to a mobile device (PDA), and to a small monitor that can be clipped to standard glasses. Therefore, the graphics generation process can be classified as resource adaptive. A great part of the resource limitations can be covered since REAL 
provides the user with information not only during the navigation task, but also in advance at a stationary information kiosk. This kiosk is able to provide sophisticated adaptation services (e.g., through the use of 3D-graphics, automatically planned camera paths, and the presentation of virtual walktroughs from a user's perspective). Both the stationary and the mobile part of the REAL system form a hybrid resource adaptive navigational aid, which provides way descriptions that are consistent over different output media [8, 1].

\section{RELATED WORK}

The CYBERGUIDE system was one of the first systems that used location aware information to help tourists [9]. Both an indoor and an outdoor navigation component were designed to assist tourists with active location sensitivity. The indoor component relied on infrared beacons broadcasting a unique ID, that was used to display an arrow on a map whenever the user entered a new room. Additionally, the user's orientation was estimated from her actual walking direction and the topology of the building. The outdoor system used GPS instead of infrared to determine the user's position and to display it on a map. Both systems operated independently from each other and could not be combined. The MARS-system [6] is an augmented reality system that provides information on the buildings on the Columbia University Campus. More recently an additional indoor component has been developed that assists the user in indoor navigational tasks [7]. The GUIDE-system is a location aware multimedia tourist guide for the City of Lancaster [3]. Based on a radio cell infrastructure adapted information is provided that is tailored to the personal preferences of the user, but also to her actual contextual situation. The DEEP MAP project [10] (carried out at the European Media Lab) focuses on a mobile tourist guide that brings together results from natural language and intelligent graphics generation. This allows a multi-modal user interface to offer the user a variety of information on the city of Heidelberg. Since GPS is used to determine the position DEEP MAP is currently restricted to outdoor scenarios. The MOBIS system [4] is a situative guide based on a PDA that provides information on the exhibits of a museum. The PDA receives its position from IR beacons distributed in the environment and uses this position as a pointer to a specific content that is stored in a database on the PDA. When comparing the IRREAL system with the MOBIS system [4] it can be observed that the same underlying technology is used for comparable tasks. While both systems depend on strong infrared senders in the environment and on infrared receivers on the mobile devices, MOBIS uses active location sensitivity and IRREAL passive location sensitivity. A similar approach is followed by the HIPS system [11]. In contrast to MOBIS a radio back-channel is used for downloading multimedia content. HIPS incorporates more powerful mobile devices (sub-notebooks), which allow for the playback of much better animations and sound files at the cost of additional weight. HIPS does not only take into account the absolute position of the user but also the relative distances to objects in the exhibition. The NEXUS system [5] aims at providing a general framework for mobile and location aware computing. The concept of an augmented world is used to store information that may be relevant to a user at a certain location. This model forms the basis for so called virtual information towers that connect information objects (i.e. from the world wide web) to real places.

Nearly all the discussed systems are designed for certain computational and communicational platforms. Either enough computational power has to be available on the mobile device (DEEP MAP), or the information has to be stored locally in a huge database (MOBIS), or has to be accessible over a network infrastructure (NEXUS, HIPS). In all three cases compromises had to be accepted between the quality and adaptivity of the presentations and security issues. It is not possible to distribute computational tasks in the environment without letting the environment know the exact user position. Another drawback of the aforementioned systems is their missing ability to adapt to the restricted cognitive resources of the user. Especially the use of mobile devices implies the need for adaptive presentation generation and interaction, given the fact that the user might be distracted or under time pressure. None of the discussed systems allows for a seamless switching from indoor (passive location sensitivity) to outdoor (active location sensitivity) scenarios and vice versa.

\section{REAL: A RESOURCE-ADAPTIVE PEDESTRIAN NAVIGA- TION SYSTEM}

Car navigation systems can be considered state of the art for luxury cars, and are now becoming more affordable to a broader range of users. These systems are widely accepted because of their simple and well designed interfaces for the specific task of providing multi-modal (graphics, text and speech) route description to a car driver. Since cars usually travel on roads, the navigation system has reasonably good visibility of the GPS satellites. In combination with compass readings, odometric information, and assuming that changes of directions are slow, the system can rely on relatively robust information on the car's location. Since the user context does not vary much either (fixed seating position, user looking in driving direction), car navigation systems are able to provide well designed incremental way descriptions (with graphics and audio). In contrast to car navigation systems the design of a pedestrian navigation system is much more complex. The main reason is that the user context is changing constantly. On the one hand the sensors' readings are not as reliable and depend on the user's location. On the other hand the supported tasks can be much more complex than in the case of the car navigation system. For example, there is a big difference between a way description for a business man who needs to be guided to the train station under time pressure and the way description for a tourist who wants to know about interesting sights in her vicinity (including the train station). In addition, pedestrian navigation systems should 
be able to operate indoor (e.g., in a museum) and outdoor (e. g., in a pedestrian area) to support these tasks. A switch between both operational modes should be as seamless as possible. The following sections address these problems by describing the pedestrian navigation system REAL and its subcomponents IRREAL and ARREAL.

\section{The Indoor Navigation System IRREAL}

For navigation inside buildings we have developed the IRREAL subcomponent, where we concentrated on a smart but simple solution: in order to relieve the load on the user's end devices as much intelligence as possible was transfered to the environment. IRREAL incorporates handheld computers (using PalmOS or WindowsCE), since these devices offer a reasonable compromise between display size and weight. Data is transmitted to the handheld using infrared because this technology also allows simultaneously for the determination of the user's current position and orientation in the environment. As most of the handheld devices are equipped with an integrated IR port, there is no need for additional hardware. The integrated IR port uses the IrDa protocol, which supports bidirectional links between IrDa devices up to a distance of two meters. Inside buildings, where we have to mount transmitters at the ceiling or at pillars we cannot use this kind of bidirectional communication. To solve this problem we have developed strong infrared transmitters that cover a range of up to twenty meters [2]. A central server constantly provides these transmitters with data, which allows for the transmission of dynamic information, e. g., bus schedules. The long distance between transmitters and receivers necessitates a special transmission protocol. The protocol enables unidirectional links between transmitters and passively receiving devices. This is one of the main differences between IRREAL and related systems in the field of mobile pedestrian navigation, where special hardware additions on the client side are required to establish a bidirectional communication (see [14] and [15]). The technology used in IRREAL to realize interactivity over unidirectional links resembles the technology known from teletext, where all information is transmitted in cycles over and over again. In contrast to teletext IRREAL transmits interactive texts and graphics, which are much more like hypertext documents. This enables a user to interact with the transmitted presentations, although there is no bidirectional connection at any time. Figure 2 shows a partial presentation graph for an incremental route description. The arrows in the figure symbolize possible user interactions with the presentation. The overlapping parts in the presentation tree represent changes of the presentation over time, which are independent from user interactions such as moving through the building. A presentation tree consists of nodes that contain text and graphics. Navigation between nodes is accomplished by clicking on interactive areas (hot-spots) that connect two nodes in the presentation tree.

It is important to stress that the environment cannot track the
A)

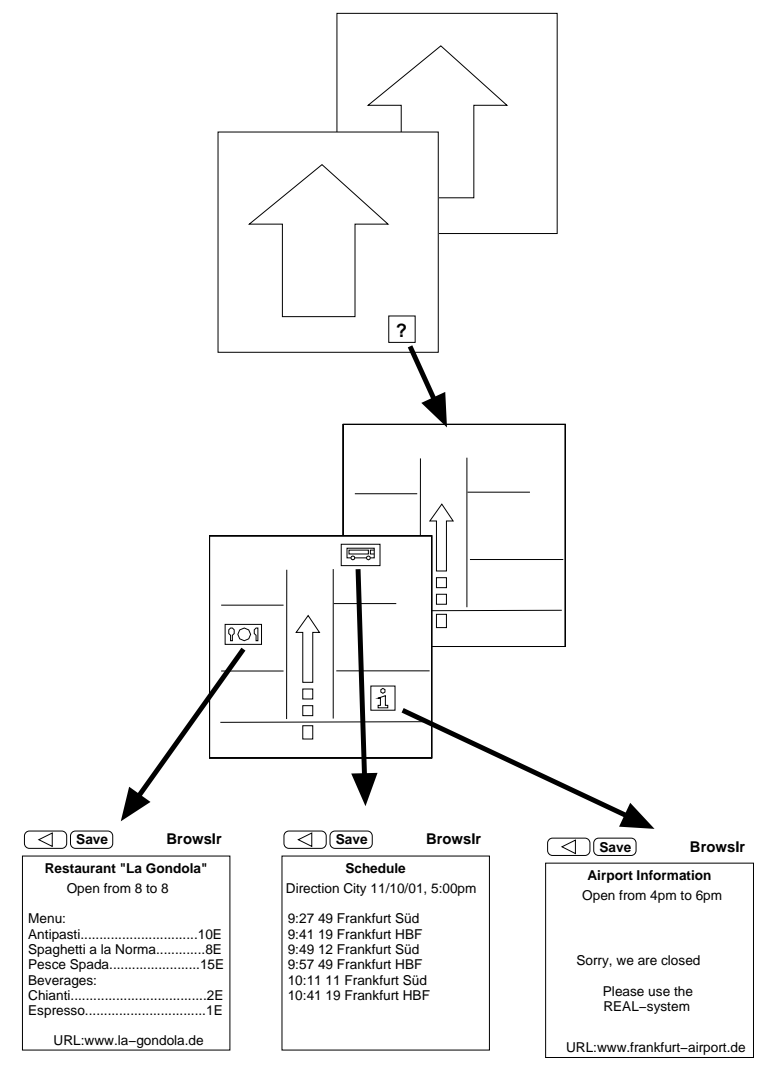

Figure 2: A presentation graph used by IRREAL

user's position at any time since the presentations are broadcasted only in one direction. This implies that all the presentations have to be transmitted in cycles at every sender all the time. Nevertheless, usability reasons force the underlying broadcast protocol to ensure that the response time for the first presentation is not too long. Therefore, instead of transmitting every node of a presentation at the same rate, we discriminate between important and less important nodes, and transmit the important ones more frequently. To achieve this we assume that every element in the $i$-th presentation layer has the same transmission probability $w_{i}$, which can be computed as follows: First of all, we determine the absolute weight of a presentation element from the $i$-th layer $w_{i}^{\prime}$ according a suitable parameter $c$ :

$$
w_{i}^{\prime}=\frac{1}{c^{i+1}}, c \geq 1
$$

The absolute weight represents the importance of a presentation element and is used to ascertain the transmission probability for the element. This is done by normalizing over the sum of all absolute weights $\left(n_{i}\right.$ stands for the number of all presentation elements in the $i$-th presentation layer).

$$
S=\sum_{i} n_{i} w_{i}^{\prime}, \quad w_{i}=\frac{w_{i}^{\prime}}{S}
$$

Assigning transmission probabilities in the aforementioned manner gives preferential treatment to nodes near the root of 
the presentation tree. In contrast terminal nodes in the presentation tree will be transmitted sparsely. Since the device is constantly receiving data and the user needs a certain amount of time to interact with the presentation, it is possible to conceal the loading time of the less important presentation parts by the interaction time of the user with the handheld. The example shown in Figure 2 illustrates this: the first item the user will see on the display is an arrow pointing in the walking direction. If she stays a little longer in the area of the transmitter, the presentation will be augmented with a help button (see Figure 2A). If the button is pushed a greater portion of the actual path will be displayed (see Figure 2B). After staying in the range of the transmitter for a while, background information about the current environment will be added to the presentation (see Figure 2C).

The unidirectional transmission protocol also enables the system to adapt to the current user's walking speed. If the user stays in a transmission area for a short time, the device normally receives high priority information first, e.g. graphical walking directions. The more time the user spends in an area the more complex the information about the environment becomes available. This allows the user to control the amount of detail of the presentation by simply waiting close to a transmitter. The addition of a concept of user groups

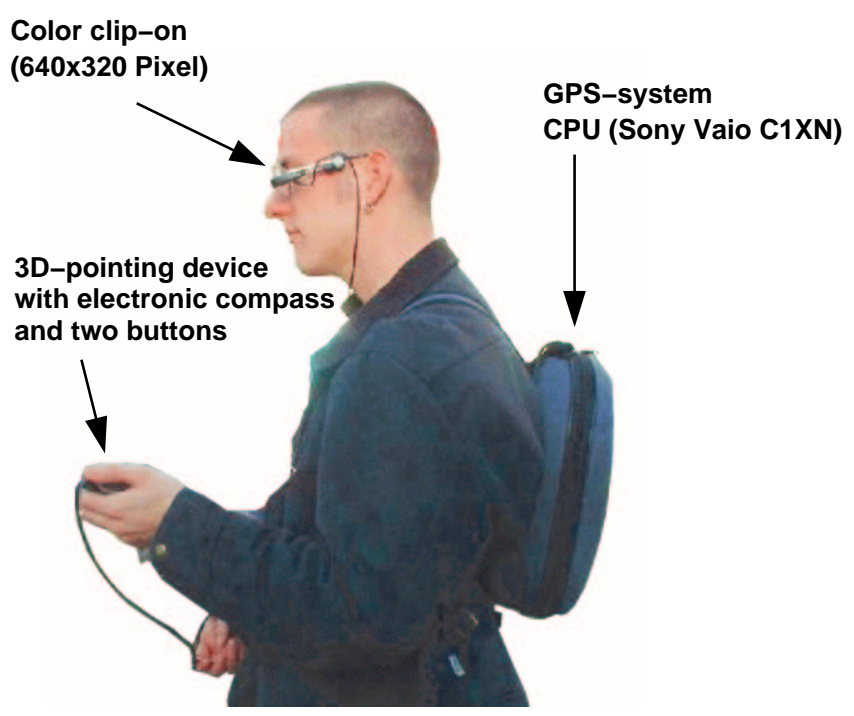

Figure 3: The components of the ARREAL system

allows the system to always comply with certain fixed constraints. For example, it is possible for a user to choose between a detailed or a more abstract type of description. These different kinds of descriptions are assigned to different user groups. The device filters the stream of data it receives, and makes available only the information relevant to the current user group. The concept of user groups is technically realized through a special kind of hot-spot. Interacting with such a special hot-spot causes a change of the user group. Since all the transmitted information packages belong to a certain user group, it is now possible for the handheld to filter the information according to the user's preferences. Of course, realizing different user groups implies to prepare different presentations for every user group.

If the user enters an area covered by a new transmitter the device will detect this change. In the new area the device will wait for the root node of the presentation tree, and will then visualize the information contained therein. This modus operandi may lead to incoherent presentations in situations, where the former transmitter streams detailed information about the environment (for instance in textual form), and the new one presents more abstract presentations (e.g., an overview map). To address this problem all the presentation trees for the transmitters should have the same logical structure, which is realized by a central planning component (see Figure 1). First of all the logical structure for all the presentations is planned and then assigned to the different transmitters. Afterwards the specific contents for each sender (texts or graphics) are filled in. The user group concept allows to define a global presentation layer for all the infrared transmitters involved in a presentation. For example, it is possible to assign a certain user group to overview maps and another to detailed instructions. In these cases the visualization remains in the same presentation layer even when the user leaves a transmission area and enters a new one. This approach ensures the overall generation of coherent presentations.

\section{The Outdoor Navigation System ARREAL}

In the ARREAL project we have been working on the development of a navigation system for pedestrians in an outdoor scenario. The scenario imposes special demands on the hardware. All components should be light, small, and unobtrusive. The ARREAL system consists of four components. The major component is a sub-notebook (Sony Vaio C1XN), used for all the relevant computations. The user's position and orientation in the environment is determined through the use of a small GPS-System (Gramin eTrax Summit) and an electronic compass (CyberTrackII from General Reality). These devices are connected to the notebook by serial ports. For graphical and textual presentations we use a special clip-on for glasses (from MicroOptical). The user may hold the electronic compass in her hands or attach it to her belt. Two additional buttons at the top of the electronic compass can be used to interact with the system analogously to a standard two button computer mouse. The modified compass can be used as a 3D-pointing device. This allows the user to retrieve additional information by simply pointing at a building. The central processing unit is carried in a small backpack (see Figure 3). The small clip-on-display (with a resolution of $640 \times 320$ Pixel) allows only for the visualization of sketch-like graphics from birds-eye or user-perspective. While overview maps are used to visualize the user's current position in the environment, graphics from the user's perspective are used to present more detailed information about the environment, e. g., information about buildings in the current line of sight. In addition, the system supports different 


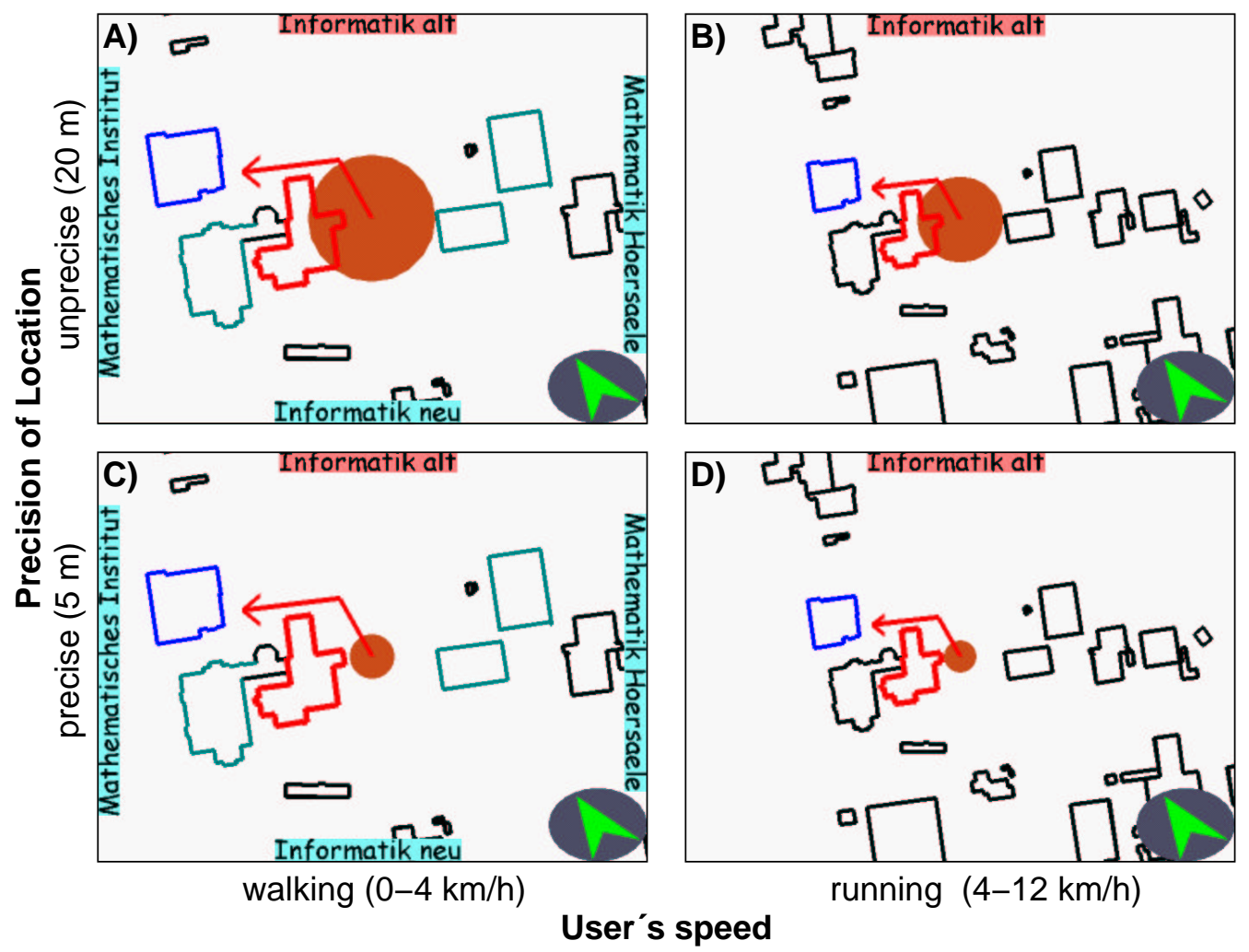

Figure 4: Adaptation of the graphical output according to the user's moving speed and the precision of location information

levels of detail in the visualization. On one hand the system is able to visualize different portions of a map or may change from an overview to a detailed view of the environment. On the other hand textual or graphical annotations can be inserted such as the names of streets or buildings (see Figure 4). Navigational instructions are given by means of arrows and spoken directions that indicate turns to the user. ARREAL reacts to the changing quality of positional and orientation information in different ways. First of all, the system chooses between two visualization modes: a birds-eye view or a userperspective. The user view only makes sense, if the system has adequate positional and orientation information to display pictures of buildings in the current line of sight. In case that positional and orientation information is only available at low quality, ARREAL prefers birds-eye view. If birds-eye view is chosen the precision of the positional information is encoded by the size of the dot, which represents the user's current position in the environment on the map. Decreasing locational information results in a bigger dot. In addition, the system takes into account the user's current walking speed. If she moves fast, the system presents a greater portion of the map in order to help the user to orientate herself. At the same time the amount of information about buildings at the edges of the display is reduced. Since textual annotations at the edges of the display serve as menu-items, the system thereby also reduces the number of possibilities to interact with the system. Information about the user's walking speed and quality of positional information taken from the GPS is used to control the level of detail by modifying the scale of the map. Information about the user's orientation is measured by the electronic compass, and is used to rotate the map to coincide with the user's view on the environment. Figure 4 shows several snapshots taken from the system's graphical output. They illustrate the system's reaction to changing precision of positional and orientation information as well as the user's speed. Figure 4A) presents the system's output for a slowly moving user and unprecise positional information, whereas Figure 4D) shows the results for exact positional information at a higher speed.

\section{Transition between IRREAL and ARREAL}

In order to ensure a seamless transition between IRREAL (indoor) and ARREAL (outdoor) several prerequisites must be fulfilled. First of all the generation processes and data for the two systems have to be coordinated. This implies that IRREAL and ARREAL have to use the same knowledge base of presentation techniques and overlapping models of the spatial environment. The indoor system IRREAL uses a 3D-model of a building's interior, whereas ARREAL uses map-like material, where buildings are represented as single spatial entities. Leaving or entering a building leads to a change of the environmental spatial representation. A particularity of the combined use of the IRREAL and ARREAL systems is the seamless switch between passive and 
active location sensitivity. If the mobile system receives data over an infrared stream, the information will be displayed on the handheld or the clip-on display (passive location sensitivity). The generated navigational instructions are adapted to the display capabilities of the device (see Figure 5). Figure

\section{A)}

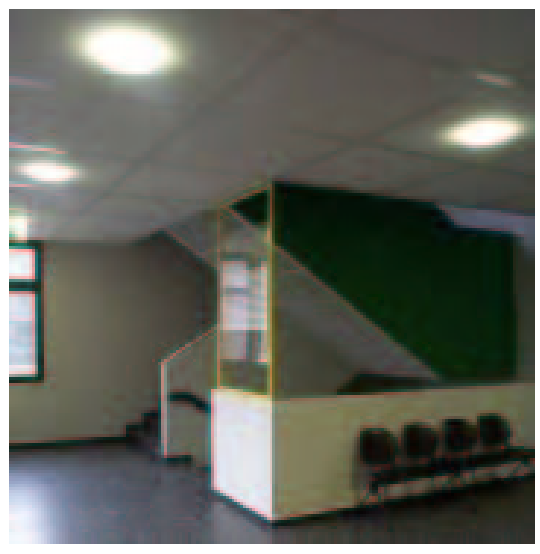

B)

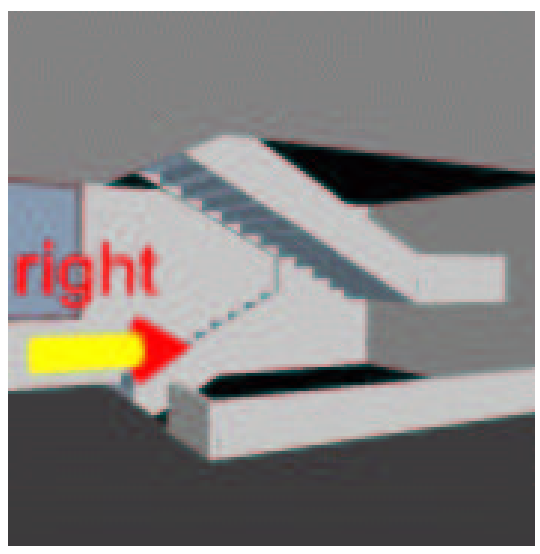

C)

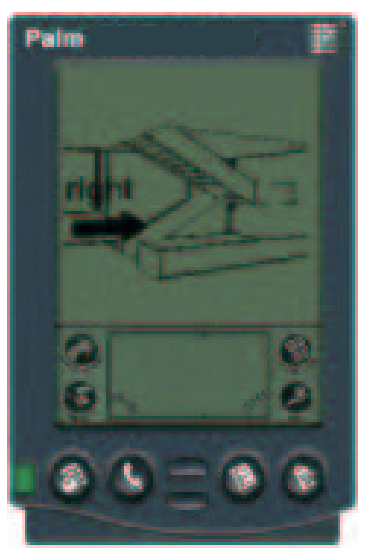

Figure 5: Graphical adaptation to different output media

5B) shows a detailed graphic for the clip-on display with a high resolution. The black and white sketch with lower resolution is generated for the handheld (Figure 5C). While the graphics generation process yields less sophisticated presentations for the handheld, these may contain more complex interactive areas than the ones generated for the use with the clip-on display. This is due to the fact that interaction with the handheld is easier than with the clip-on display. The ability to generate graphics for different output media is realized through the use of a central presentation server. If the user of ARREAL leaves the building, the mobile system uses active location sensitivity to determine the user's position in the environment with the help of GPS satellites. The user's position is reported to the server and the presentation is adapted dynamically.

\section{CONCLUSION AND FUTURE WORK}

The work presented in this paper describes how a hybrid pedestrian navigation system can tailor its presentations to limited technical resources of the device and the limited cognitive resources of the user. All the techniques that were introduced have been implemented in a running prototype. The IRREAL system has been tested extensively in our computer science department, the structure and content of presentations have been generated automatically by our presentation server. The outdoor component ARREAL has also been tested in the outdoor area of Saarland University. Figure 6 summarizes the limited resources taken into account by the system and gives an overview about the resulting adaptation capabilities. Another major contribution of the two systems is the resource adaptive combination of active and passive location sensitivity, where the change of the adaptation paradigm remains unnoticed by the user. In the near future

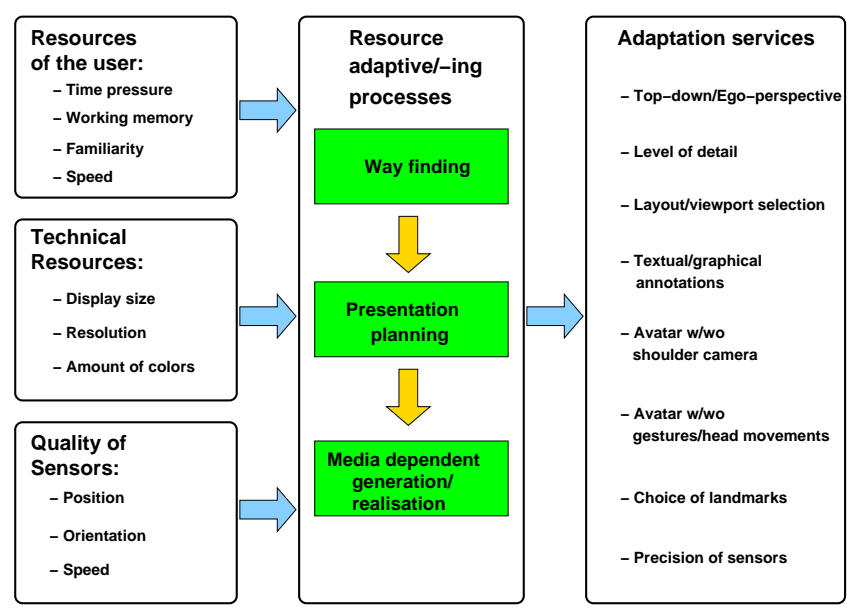

Figure 6: Typology of the considered limited resources and adaptation capacities

we will transfer parts of the system to an instrumented environment. Until now, only the user's position, orientation, and speed are taken into account. In the future we plan to observe the user's interactions with virtual and real objects in such an environment to improve the system's adaptation capabilities. This involves, e. g., the recognition and further support of the user's intentions and plans. 


\section{ACKNOWLEDGMENTS}

The research described in this paper was funded by 'Deutsche Forschungsgemeinschaft' DFG within their Collaborative Research Program 378 on "resource-adaptive cognitive processes".

\section{REFERENCES}

1. A. Butz, J. Baus, A. Krüger, and M. Lohse. A Hybrid Indoor Navigation System. In IUI2001: International Conference on Intelligent User Interfaces, pages 2533, New York, 2001. ACM.

2. A. Butz and A. Krüger. Orts- und richtungsabhängige Informationspräsentation auf mobilen Geräten. $I T+T I$, Oldenbourg, (2):90-96, 2001.

3. K. Cheverst, N. Davies, K. Mitchell, A. Friday, and C. Efstratiou. Developing a Context-aware Electronic Tourist Guide: Some Issues and Experiences. In Proceedings of CHI 2000, pages 17-24, 2000.

4. J. L. Encarnacao and T. Kirste. Beyond the desktop: Natural interaction and intelligent assistance for the everyday life. In Heinz Nixdorf Museumsforum, editor, Alltag der Zukunft - Informationstechnik verändert unser Leben, pages 39-57. Schöningh, Paderborn, München, Wien, Zürich, 2000.

5. F. Hohl, U. Kubach, A. Leonhardi, K. Rothermel, and M. Schwehm. Next century challenges: Nexus - an open global infrastructure for spatial-aware applications. In T. Imielinski and M. Steenstrup, editors, International Conference on Mobile Computing and Networking (MobiCom'99), pages 249-255. ACM/IEEE, ACM Press, 1999.

6. T. Höllerer, S. Feiner, T. Terauchi, G. Rashid, and D. Hallaway. Exploring mars: Developing indoor and outdoor user interfaces to a mobile augmented reality system. Computers and Graphics, 23(6):779-785, December 1999.

7. T. Höllerer, D. Hallaway, N. Tienna, and S. Feiner. Steps towards accommodating variable position tracking accuracy in a mobile augmented reality system. In IJCAI-2001 Working Notes: AI in Mobile Systems (AIMS), pages 31-37, 2001.

8. A. Krüger, J. Baus, and A. Butz. Smart Graphics in Adaptive Way Descriptions. In Proceedings of the working conference on Advanced Visual Interfaces AVI 2000, May 24-26, 2000, Palermo, ITALY. ACM press, 2000.

9. S. Long, K. Kooper, G. D. Abowd, and Atkeson C. G. Rapid Prototyping of Mobile Context-Aware Applications: The Cyberguide Case Study. In Proceedings of the 2nd ACM International Conference on Mobile Computing and Networking, pages 97-107, 1996.
10. R. Malaka and A. Zipf. Deep Map - challenging IT research in the framework of a tourist information system. In D. R. Fesenmaier, S. Klein, and D. Buhalis, editors, Information and communication technologies in tourism 2000, pages 15-27. Springer, Wien, 2000.

11. E. Not, D. Petrelli, M. Sarini, O. Stock, C. Strapparava, and M. Zancanaro. Hypernavigation in the physical space: Adapting presentations to the user and to the situational context. The New Review of Hypermedia and Multimedia, 4:33-45, 1998.

12. W. Wahlster, A. Blocher, J. Baus, E. Stopp, and H. Speiser. Ressourcenadaptierende Objektlokalisation: Sprachliche Raumbeschreibungen unter Zeitdruck. Kognitionswissenschaft, Band 7, Heft 3, pages 111-118, 1998.

13. W. Wahlster and W. Tack. SFB 378: Ressourcenadaptive Kognitive Prozesse. In Matthias Jarke, editor, Informatik '97: Informatik als Innovationsmotor, pages 51-57, Berlin, 1997. Springer.

14. R. Want, A. Hopper, V. Falcao, and J. Gibbons. The active badge location system. ACM Transactions on Information Systems, 10(1):91-102, January 1992.

15. R. Want, B. Schilit, N. Adams, R. Gold, K. Petersen, D. Goldberg, J. Ellis, and M. Weiser. An overview of the ParcTab ubiquitous computing experiment. IEEE Personal Communications, pages 28-43, December 1995.

16. H. Zimmer, H. Speiser, and J. Baus. Die Selektion dimensionaler Präpositionen: automatisch und nicht ressourcenadaptierend. Kognitionswissenschaft, Band 9, Heft 3, pages 114-121, October 2001. 